Les numéros correspondant à la pagination de la version imprimée sont placés entre crochets dans le texte et composés en gras.

\title{
Le rapport, nouveau, à la pureté et à l'impureté dans l’Évangile de Marc et les conséquences qui en résultent
}

\author{
Christian Grappe \\ Université de Strasbourg - \\ Faculté de Théologie Protestante - EA 4378 (Théologie protestante) \\ Palais Universitaire - 9, place de l'Université - F-67084 Strasbourg Cedex
}

Les numéros correspondant à la pagination de la version imprimée sont placés entre crochets dans le texte et composés en gras.

Publié in: Reading the Gospel of Mark in the Twenty-First Century: Method and Meaning. Edited by Geert Van Oyen (Bibliotheca Ephemeridum theologicarum Lovaniensium 301), Leuven, 2019, p. 115-139.

I. EN GUISE DE PRÉLIMINAIRE :

PuRETÉ ET IMPURETÉ DANS LA PENSÉE DE LA BIBLE HÉBRAÏQUE

ET DANS LE JUDAÏSME INTERTESTAMENTAIRE

La pensée de la Bible hébraïque suppose une série de distinctions et de séparations nécessaires. Elles ont été nécessaires à l'origine pour ordonner le chaos primordial ${ }^{1}$. Elles le sont quotidiennement pour que le peuple demeure fidèle à la vocation que lui a assignée son Dieu créateur et sauveur.

C'est ce qu'exprime Lv 20,24-26.

\begin{tabular}{|c|c|}
\hline Lévitique 20,24-26 (TM) & Traduction Segond (légèrement retouchée) \\
\hline 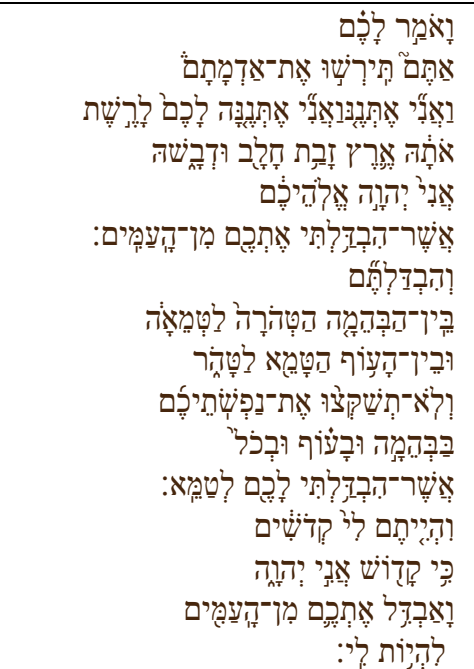 & $\begin{array}{l}{ }^{24} \text { Je vous ai dit: } \\
C^{\prime} \text { est vous qui posséderez leur pays; } \\
\text { je vous en donnerai la possession: } \\
\text { c'est un pays où coulent le lait et le miel. } \\
\text { Je suis l'Éternel, votre Dieu, } \\
\text { qui vous ai séparés des peuples. } \\
{ }^{25} \text { Vous observerez la distinction } \\
\text { entre les animaux purs et impurs, } \\
\text { entre les oiseaux impurs et purs, } \\
\text { afin de ne pas rendre vos personnes abominables } \\
\text { par des animaux, par des oiseaux, par tous les reptiles de la terre, } \\
\text { dont je vous ai appris à vous séparer comme impurs. } \\
{ }^{26} \text { Vous serez saints pour moi, } \\
\text { car je suis saint, moi, l'Éternel ; } \\
\text { je vous ai séparés des peuples, } \\
\text { afin que vous soyez à moi. }\end{array}$ \\
\hline
\end{tabular}

${ }^{1}$ Voir notamment l'importance qui revient au verbe $b d l$ (séparer) dans le premier récit de la Genèse : 1,4 (premier jour : séparation de la lumière et des ténèbres) ; 1,6.7 (deuxième jour : séparation des eaux d'en haut des eaux d'en bas) ; 1,14.18 (quatrième jour : établissement des luminaires pour séparer jour et nuit et lumière et ténèbres). 
[116] C'est ce que rappelle aussi, comme l'a notamment reconnu F. Schmidt ${ }^{2}$, Lv 10,10 qui insiste sur la nécessité impérieuse qu'il y a à « être à même de distinguer ou séparer (bdl) le sacré du profane, ce qui est impur de ce qui est pur ».

De la même manière qu'étaient établies des distinctions entre les êtres, étaient effectuées des distinctions entre les animaux, selon un ordre qui gravitait autour du Sanctuaire et du culte, l'ensemble s'inscrivant dans " le grand acte liturgique qu'était la reconnaissance de Dieu et son adoration - acte qui culminait dans le sacrifice au Temple ${ }^{3}$.

Les distinctions nécessaires concernaient également les lieux ${ }^{4}$ et les temps puisque certains de ces temps étaient mis à part et requéraient des cérémoniels particuliers (sabbat ; fêtes de pèlerinage.... $)^{5}$.

Le système ainsi élaboré gravitait autour du sanctuaire.

Jacob Milgrom 6 a montré qu'il repose sur les données fondamentales suivantes : les personnes et les objets sont susceptibles de se trouver dans quatre états : saint (holy) ; profane (common), pur (pure) et impur (impure). Deux d'entre eux peuvent exister simultanément : saint ou profane, pur ou impur. De plus, une combinaison est exclue dans le système sacerdotal : alors que le profane peut être soit pur soit impur, le sacré $(\text { sacred })^{7}$ ne peut jamais être impur (...). Par exemple, le laïc (profane) est censé être pur, sauf s'il est pollué par quelque impureté, comme un cadavre d'animal [117] (carcass) (Lv 11), la lèpre (Lv13-14), un écoulement génital (Lv $12 ; 15$ ) ou un cadavre humain (corpse) (Nb 19), cas pour lesquels des procédures de purification sont prescrites. II n'y a ni danger ni responsabilité pour le laïc qui contracte une impureté tant qu'il ne lui permet pas de se prolonger. II n'en va pas de même pour le sacré ${ }^{8}$. Le sanctuaire, par exemple, doit rester en tout temps pur ; l'impureté qui l'affecte doit être immédiatement purgée, de peur que la communauté tout entière ne soit mise en péril (blighted).

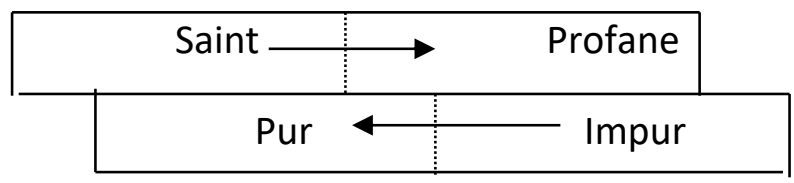

Ces relations sont dépeintes dans le diagramme ci-dessus ${ }^{9}$. Le profane est contigu aux

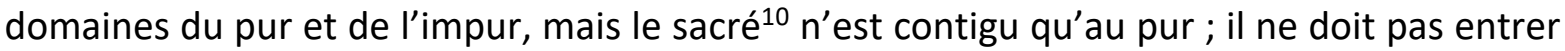
en contact avec l'impur. Qui plus est, la transformation du saint en profane n'est pas toujours

[116] ${ }^{2}$ F. Schmidt, La pensée du Temple. De Jérusalem à Qoumrân. Identité et lien social dans le judaïsme ancien (La Librairie du XX ${ }^{\mathrm{e}}$ siècle), Paris, Seuil, 1994, p. 81 (et passim).

${ }^{3}$ M. Douglas, De la souillure. Essai sur les notions de pollution et de tabou, Paris, 1981, 76.

4 Voir le système mishnique de pureté, mais aussi les degrés de pureté envisagés dans le Rouleau du Temple ; l'organisation du camp du désert ; du Temple aux douze portes ou de la Communauté-sanctuaire de Qumrân. Sur tout cela, ScHMIDT, La pensée du Temple (n. 2), pp. 125-235.

${ }^{5}$ Suivant la séquence des traités du deuxième ordre de la Mishna (Mo'ed [jours de fête]), J.H. NEYREY, The Idea of Purity in Mark's Gospel, in Semeia 35, 1986, 99, propose ainsi que l'on ait distingué, par ordre d'importance : 1 . Sabbat et jonctions sabbatiques (Erubin) ; 2. Pesahim ; 3. Yoma ; 4. Sukkoth ; 5. Yom Tob (jour férié) ; 6. Rosh ha-shana ; 7. Ta'anit (le jeûne) ; 8. Megilla (le rouleau, plus particulièrement celui d'Esther, lu lors de la fête de Pourim) ; 9. Mo'ed qatan (fêtes de seconde importance). Il n'est toutefois nullement certain que cette séquence dénote toujours un tel ordre d'importance, d'autant qu'aucun traité n'est consacré à la fête des Semaines et que le dernier, Hagiga (célébration), aborde en bloc les fêtes de pèlerinage dont on ne saurait dire qu'elles soient les moins notables.

6 Voir notamment J. Milgrom, Sancta Contagion and Altar/City Asylum, in J.A. Emerton (éd.),: Congress Volume. Vienne 1980 (SupplVT 32), Leiden, Brill, 1981, 278-310 ; ID., Leviticus 1-16. A New Translation with Introduction and Commentary (AncB 3), New York, Doubleday, 1991; ID., Priestly ("P") Source, in D.N. FreEdman (éd.), The Anchor Bible Dictionary. Volume 5. O-Sh., New York, Doubleday, 1992, 454-461.

7 Pour la cohérence du raisonnement avec le diagramme, il vaut mieux entendre ici « le saint ».

[117] ${ }^{8}$ Même remarque qu'à la note précédente.

${ }^{9}$ Nous avons cependant inversé le sens de la flèche au registre inférieur car cette inversion fait mieux apparaître que seules la sainteté et l'impureté ont, en temps normal, un pouvoir contagieux, selon la démonstration de Milgrom.

10 Même remarque qu'à la note précédente. 
nécessairement illégitime (" désécration »), requérant alors une offrande de réparation et une amende. Elle peut être parfaitement légitime ("désanctification »), requérant alors seulement une surcharge de $20 \%$ (cf. Lv 27,13-31). Réciproquement, le saint ne peut et ne doit (may) jamais devenir impur. Ces deux catégories sont antagonistes, totalement opposées. Ce sont des antonymes. De plus, ce sont des catégories dynamiques : elles cherchent à étendre leur influence et à prendre le contrôle des autres catégories, le profane et le pur. Au contraire des deux précédentes, ces deux dernières catégories sont statiques. Elles ne peuvent communiquer leur état ; il n'y a pas de pureté contagieuse ou de " profanité » (commonness) contagieuse. Ce sont, de fait, des catégories secondaires. Elles revêtent leur identité à partir de leurs antonymes. La pureté est l'absence d'impureté ; la " profanité » est l'absence de sainteté ${ }^{11}$. En conséquence, les frontières entre le saint et le profane et entre le pur et l'impur sont représentées par une ligne discontinue. II n'y a pas de frontière fixe. Israël par sa conduite peut déplacer les frontières - dans les deux sens. Mais il est appelé à n'agir que dans une direction : faire avancer le saint dans le domaine du profane et diminuer l'impur et, ainsi, accroître le domaine du pur ${ }^{12}$.

[118] Certains passages bien spécifiques et qui nous semblent revêtir une importance toute particulière pour comprendre la dynamique à l'œuvre dans le Nouveau Testament, et dans l'Évangile de Marc en particulier, attestent l'attente eschatologique d'une sainteté venant envahir la sphère profane ${ }^{13}$. Ils s'expliquent sans doute par la conviction selon laquelle la gloire divine a vocation à englober toute la terre ( $\mathrm{Nb} 14,21)$. Nous mentionnerons ici Za 14,20-21 et Jr 31,31-4014. Dans le premier de ces textes, à I'horizon dernier, toute réalité profane, des clochettes des chevaux à la marmite la plus banale, se trouve désormais consacrée et la sanctification de l'espace rend superflue la présence des intermédiaires - les marchands du Temple en l'occurrence - qui avaient rempli jusque-là une fonction d'intermédiaires obligés en vue de la célébration du culte (Za 14,20-21). Dans le second, même la vallée des cadavres et des cendres est promise à être un espace de sainteté pour le Seigneur au sein de l'inviolable ville sainte des temps derniers (Jr 31,40). La sainteté ici envisagée n'est plus seulement contagieuse ou offensive. Elle n'est plus mise en péril par l'impureté ou par la souillure. Elle prévaut. Elle est victorieuse, triomphante, phénomène que nous rencontrerons aussi dans l'Évangile de Marc.

Que le système du pur et de l'impur soit lourd d'enjeu et ait fait l'objet de débat dès avant le Nouveau Testament est attesté par 4QMMT (4QMiqsat Ma'asei ha-Torah ou Lettre halakhique), document retrouvé à l'état fragmentaire à Qumrân (4Q394-399) ${ }^{15}$ et qui remonte au deuxième siècle avant notre ère. Les termes d'une polémique opposant les

11 Ainsi W. PASCHEN, Rein und Unrein. Untersuchung zur biblischen Wortgeschichte (StANT 24), München, Kösel Verlag, 1970, p. 64).

12 J. MILGROM, Leviticus 1-16 (n. 6), p. 732 (voir déjà p. 616-617).

[118] ${ }^{13}$ Ainsi déjà L. Goppelt, Theologie des Neuen Testaments. Erster Teil. Jesu Wirken in seiner theologischen Bedeutung, herausgegeben von J. RolofF, Göttingen, Vandenhoeck \& Ruprecht, 1975, p. 142, K. PAESLER, Das Tempelwort Jesu. Die Traditionen von Tempelzerstörung im Neuen Testament (FRLANT184), Göttingen, Vandenhoeck \& Ruprecht, 1999, p. 247 ; K.W. WEYDE, The Appointed Festivals of YHWH. The Festival Calendar in Leviticus 23 and the sukkôt Festival in Other Biblical Texts (FAT 2. Reihe 4), Tübingen, Mohr Siebeck, 2004, p. 229-236. T. HolmÉn, Jesus and the Purity Paradigm, in Handbook for the Study of the Historical Jesus. Volume 3. The Historical Jesus, Leiden - Boston, Brill, 2011, pp. 2720-2725, fait valoir que le modèle traditionnel est illustré par Ag 2,11-13, mais il ne prend pas en compte les textes que nous produisons ici, ce qui l'empêche, de notre point de vue, d'évaluer pleinement la portée de ce qu'il appelle pourtant la vision eschatologique de la pureté de Jésus, une vision dont il reconnaît qu'elle permet dès à présent à ceux qui sont rituellement impurs de devenir partie intégrante du Royaume de Dieu, cela du fait que la pureté de Jésus est transférée à l'impur sans recours aux voies traditionnelles de purification (p. 2743).

38.

14 WEYDE, Appointed Festivals (n. 13), p. 231-231, mentionne ces deux textes et y associe Ex 28,36-

15 A. CAQuot, Un exposé polémique de pratiques sectaires (4Q MMT), in RHPR 76, 1996, 257-276, a proposé la première traduction française de ce document. 
membres de la secte essénienne en voie de constitution à une autorité exerçant sans doute le contrôle sur le culte au Temple, selon toute vraisemblance un grand prêtre hasmonéen en exercice, y sont consignés. Ce dernier est invité, de manière [119] encore courtoise, à rejoindre le point de vue des dissidents, comme l'atteste la finale de l'écrit (4Q398, fragments 14-17, 2,1-7 [= 4QMMT C 26-32]).

4Q398, fragments 14-17, 2,2-7 (= 4QMMT C 27-32)

"Nous t'avons aussi écrit ${ }^{3}$ quelques-unes des pratiques de la Loi (מקצת מעשי התורה) que nous avons estimées bonnes pour toi et pour les tiens, car nous avons vu que ${ }^{4}$ tu possèdes intelligence et connaissance de la Loi. Examine tout cela et demande-Lui de diriger ${ }^{5}$ ta décision et d'éloigner de toi (toute) pensée mauvaise et toute décision de Bélial, ${ }^{6}$ afin que tu sois joyeux à la fin des temps, parce que tu auras trouvé que quelques-uns de nos propos (מקצת דَברינו) (sont) corrects. ${ }^{7}$ Et cela te sera compté comme justice, parce que tu auras fait ce qui est bon et droit devant Lui, pour ton bien et (celui) d'Israël ${ }^{16}$.

L'analyse du document permet de constater que la polémique ainsi menée s'apparente en fait à celle qui, selon la Mishna, opposa ultérieurement sadducéens et pharisiens, le grand prêtre visé défendant un point de vue proche de celui que prônèrent plus tard ces derniers ${ }^{17}$. Elle a trait à des dispositions bien précises, qui touchent à des questions de pureté et qui donnent lieu à divergence de vues. L'écrit défend une position rigoriste face à ce qui est tenu pour le laxisme des autorités en place, et ce sur des questions fort diverses ${ }^{18}$. II le fait en des termes (" voici quelques-uns de nos articles " : אלה מקצת דברינו [4Q394, fragments 3-7, colonne $1,4)$ qui évoquent déjà le point de vue d'une école ou d'un parti. Ajoutons ici que ces termes ne sont pas sans trouver d'écho dans d'autres que l'on rencontre ultérieurement dans la littérature rabbinique ${ }^{19}$, héritière pourtant du point de vue des adversaires combattus par la lettre halakhique!

[120] Ainsi se trouve démontrée l'ancienneté des débats relatifs aux questions de pureté au sein du judaïsme intertestamentaire et aussi de bien des dispositions pharisiennes que l'on ne trouve consignées qu'à date beaucoup plus tardive dans la littérature rabbinique $^{20}$. Cela dit, le système lui-même a donné lieu à des interprétations diverses du fait que deux modèles au moins coexistent au sein du Pentateuque en ce qui concerne l'extension de la sainteté et l'intensité de l'observance nécessaire des règles de pureté qui

[119] ${ }^{16}$ Traduction empruntée à Ibid., p. 274.

17 Ainsi L.H. Schiffman, "Miqtsat Ma'asei ha-Torah", in L.H. Schiffman - J.C. VAnderKam (éds), Encyclopedia of the Dead Sea Scrolls. Volume 1, Oxford, University Press, 2000, p. 559.

18 Sont refusés notamment l'introduction de blé des Gentils dans le sanctuaire (B,3b-5, attesté en $4 Q 394$, fr. 3-7, 1), la consommation nocturne de l'offrande végétale accompagnant un sacrifice pacifique (B,10-13a, attesté en 4Q394, fr. 3-7, col. $1+4 Q 395$, col. 1), la pratique consistant à ne pas attendre que le soleil se soit couché sur l'impureté de celui qui s'apprête à préparer l'eau lustrale à partir des cendres de la vache rousse (B,13b-17, attesté en 4Q394, fr. 3-7, col. 1 et $2+4 Q 395$, col. 1$)$, tout abattage profane à proximité du Temple (B,27b-35, attesté en 4Q394, fr. 3-7, col. 1 et $2+4 Q 397$, col. 1-2), toute forme de mariage mixte (B,39-49a, attesté en 4Q396, fr. 1-2, col. $1+4 Q 394$, fr. 8, col. 3 et 4Q397, fr. 5), le point de vue selon lequel un liquide transvasé pourrait n'être pas vecteur d'impureté (D,55-58a, attesté en 4Q394, fr. 8, col. 4), l'introduction de chiens à Jérusalem (B,58b-62a, attesté en 4Q394, fr. 6, col. 4+4Q397, fr. 6-13), l'admission avant terme du lépreux à la «pureté sacrée » (B,64b-72a, attesté en 4Q396, fr. 1-2, col. 3-4 + 4Q397, fr. 6-13), tout mariage entre un prêtre et une laïque (B,75-82, attesté en 4Q396, fr. 1-2, col. 4).

${ }_{19}$ CAQUOT, in $R H P R$ 76, 1996 (n. 15), p. 261, rapproche ainsi la formule que nous avons reproduite de celle que l'on rencontre en Tosefta Shabbat 1,16: «Voici (quelques-unes) des règles que l'on a énoncées dans le cénacle de R. Hanania b. Hezeqyah ».

[120] ${ }^{20}$ Ainsi, notamment, L.H. SchifFman, "The Temple Scroll and the Systems of Jewish Law of the Second Temple Period", in : Temple Scroll Studies. Papers presented at the International Symposium on the Temple Scroll. Manchester, December 1987 (JSPE.S 7), Sheffield, JSOT Press, 1989, 239-255 ; M. HENGEL - R. DEINES, E. P. Sanders' 'Common Judaism', Judaism, and the Pharisees, in The Journal of Theological Studies 46, 1995, p. 8 ; H.K. HARRInGton, "Biblical Law at Qumran", in P.W. FlinT - J.C. VANDERKAM, The Dead Sea Scrolls after Fifty Years. A Comprehensive Assessment, Leiden, Brill, 1998, 160-185. 
doit en résulter : le modèle de $P$, qui limite la sainteté au sanctuaire et aux seuls prêtres ; le modèle de $H$, qui l'étend, à partir du Temple, à l'ensemble de la Terre Promise et à tous ceux qui y résident ${ }^{21}$.

Le système mishnique de l'impureté 22 et de la pureté ${ }^{23}$ tel que nous permet de le reconstituer le traité Kelim de la Mishna fournit un aperçu de la carte de la pureté telle qu'elle se fixa en milieu pharisien. Même si ce traité ne remonte, sous sa forme écrite, qu'au deuxième siècle de notre ère, l'importance qu'il accorde au sanctuaire nous renvoie assurément à des représentations antérieures et illustre combien la pensée du Temple était prégnante en milieu pharisien.

\begin{tabular}{|c|c|}
\hline \multicolumn{2}{|r|}{ Les 10 degrés de consécration selon Mishna Kelim 1,6-8 ${ }^{24}$} \\
\hline 1 & $\begin{array}{l}\text { 6. Il y a dix degrés de consécration : la terre d'Israël est la plus sainte de toutes les terres. En quoi } \\
\text { consiste sa sainteté ? En ce qu'on tire d'elle la gerbe, les prémices, les deux pains, qu'on ne tire pas } \\
\text { des autres terres. }\end{array}$ \\
\hline 2 & $\begin{array}{l}\text { 7. Plus sainte qu'elle sont les villes entourées d'un rempart, parce qu'on en chasse les lépreux et } \\
\text { qu'on y promène autant qu'on veut les morts; mais une fois sortis, on ne peut les retourner. }\end{array}$ \\
\hline 3 & $\begin{array}{l}\text { Plus saint encore est l'intérieur du rempart (de Jérusalem) : car on y mange les saintetés inférieures } \\
\text { et la dîme seconde; }\end{array}$ \\
\hline [121] 4 & $\begin{array}{l}\text { Plus sainte est la montagne de la maison [du Temple], parce que ne peuvent pas y entrer ceux et } \\
\text { celles qui ont des écoulements, des menstruées et des accouchées. }\end{array}$ \\
\hline 5 & $\begin{array}{l}\text { Plus saint encore est le Hél (forteresse : partie du Temple), parce que ne peuvent pas y entrer des } \\
\text { idolâtres, des gens impurs par suite d'une mort ; }\end{array}$ \\
\hline 6 & $\begin{array}{l}\text { Plus saint encore est le parvis des femmes, parce que ne peut y entrer celui qui s'est baigné dans la } \\
\text { journée, mais les contrevenants ne sont pas tenus au sacrifice. }\end{array}$ \\
\hline 7 & $\begin{array}{l}\text { Plus saint encore est le parvis des Israélites parce que n'y entre pas celui qui a besoin d'expiation et } \\
\text { qui y contrevient doit offrir un sacrifice ; }\end{array}$ \\
\hline 8 & $\begin{array}{l}\text { Plus saint encore est le parvis des prêtres puisque les Israélites n'y entrent pas, sauf quand ils sont } \\
\text { obligés à imposer les mains, immoler et balancer. }\end{array}$ \\
\hline [9] & $\begin{array}{l}\text { Plus saint encore est l'espace entre l'autel et le porche (le vestibule), parce que n'y entrent pas les } \\
\text { prêtres affectés d'une tare ou les cheveux en désordre. }\end{array}$ \\
\hline 9 & $\begin{array}{l}\text { Plus saint encore est le sanctuaire parce que n'y entrent que les prêtres, qui se sont lavés mains et } \\
\text { pieds. }\end{array}$ \\
\hline 10 & $\begin{array}{l}\text { 8. Plus saint encore est le saint des saints, parce que n'y entre que le grand prêtre, au jour de } \\
\text { l'Expiation et pour le culte. }\end{array}$ \\
\hline & $\begin{array}{l}\text { Suivant R. Yosé, l'espace entre l'autel et le porche est égal au sanctuaire, parce que n'y entrent pas } \\
\text { les prêtres affectés de tare, aux cheveux en désordre, ayant bu du vin et qui ne se sont pas lavé } \\
\text { mains et pieds; i et on s'écarte de cet espace à l'heure de l'encens. }\end{array}$ \\
\hline
\end{tabular}

\begin{tabular}{|l|l|l|}
\hline \multicolumn{4}{|c|}{ Explication des degrés de consécration en fonction de leur niveau croissant de pureté } \\
\hline 1 & $\begin{array}{l}\text { La Terre d'Israël (plus sainte } \\
\text { que les autres terres) }\end{array}$ & Produit la gerbe, les prémices, les deux pains \\
\hline 2 & $\begin{array}{l}\text { Villes entourées d'un rempart } \\
\text { Intérieur des remparts de } \\
\text { Jérusalem }\end{array}$ & On y mange les saintetés inférieures et la seconde dîme \\
\hline
\end{tabular}

${ }^{21}$ Ainsi Jacob Milgrom, dans nombre de ses publications, parmi lesquelles nous citerons ici : «The Qumran Cult: Its Exegetical Principles », in G. BRooKE (éd.), Temple Scroll Studies. Papers presented at the International Symposium on the Temple Scroll. Manchester, December 1987, Sheffield, Sheffield Academic Press, 1989, pp. 167-168 ; ID., " Priestly ("P") Source », in D.N. Freedman (éd.), The Anchor Bible Dictionary. Volume 5. O-Sh, New York, Doubleday, 1992, p. 457.

${ }_{22}$ Mishna Kelim I,1-4.

${ }^{23}$ Mishna Kelim I,6-8.

24 Traduction empruntée à J. BonsIRVEN, Texte rabbiniques des deux premiers siècles chrétiens pour servir à l'intelligence du Nouveau Testament, Roma, Pontificio Istituto Biblico, 1955, p. 656 [n² 2344 ]. 


\begin{tabular}{|l|l|l|}
\hline 4 & Montagne de la Maison & $\begin{array}{l}\text { N'y accèdent ni celle ou celui qui est atteint d'écoulement, ni celle } \\
\text { qui a ses menstrues, ni la femme accouchée }\end{array}$ \\
\hline [122] 5 & $\begin{array}{l}\text { Hèl (forteresse : partie du } \\
\text { Temple) }\end{array}$ & $\begin{array}{l}\text { N'y accèdent ni les idolâtres, ni les gens contaminés par un } \\
\text { cadavre }\end{array}$ \\
\hline 6 & Parvis des femmes & $\begin{array}{l}\text { N'y accède pas celle ou celui qui a le statut de tebul yom (c'est-à- } \\
\text { dire qui a pris son bain de purification dans la journée et doit } \\
\text { attendre le soir pour être totalement pur) }\end{array}$ \\
\hline 7 & Parvis des Israélites & N'y accède pas celui qui a besoin d'expiation \\
\hline 8 & Parvis des prêtres & $\begin{array}{l}\text { N'y accèdent pas les lsraélites, sauf quand ils sont tenus d'imposer } \\
\text { les mains, d'immoler ou de balancer (la gerbe) }\end{array}$ \\
\hline $9 a$ & $\begin{array}{l}\text { Espace entre l'autel et le } \\
\text { porche }\end{array}$ & $\begin{array}{l}\text { N'y accède pas le prêtre affecté d'une tare ou ayant les cheveux } \\
\text { en désordre }\end{array}$ \\
\hline $9 b$ & $\begin{array}{l}\text { Sanctuaire } \\
\text { 10. Saint des Saints }\end{array}$ & $\begin{array}{l}\text { N'y accèdent que les prêtres qui se sont lavé mains et pieds } \\
\text { N'y accède que le grand prêtre le jour des Expiations }\end{array}$ \\
\hline
\end{tabular}

De son côté, l'attitude propre des esséniens peut être mieux comprise, comme l'a montré F. Garcia-Martinez, à partir d'une prise en compte du Rouleau du Temple ${ }^{25}$. Cet écrit illustre une triple tendance :

- « tendance à élargir à toute la ville sainte le niveau de pureté propre au Temple $»^{26}$;

- " tendance à élargir à tout le peuple les normes de pureté spécifiques aux prêtres ${ }^{27}$;

- « tendance à élargir et à radicaliser le champ de contamination ${ }^{28}$.

[123] N'ayant pas réussi à imposer leur point de vue et convaincus par ailleurs de la souillure du sanctuaire, les esséniens furent conduits à la rupture. Et ce qui peut frapper, dans les écrits assurément consécutifs à cette rupture, tels l'Écrit de Damas et la Règle de la Communauté, c'est «le transfert du problème de la pureté de la sphère du Temple à la sphère de la communauté elle-même : l'impureté qui interdisait à l'origine d'approcher le Temple aboutit maintenant à l'interdiction d'approcher la communauté $"{ }^{29}$. En deux endroits dans l'Écrit de Damas, il est question ainsi du coupable qui "sera séparé de la tohorah ", c'est-à-dire de la nourriture pure de la Communauté (CD 9,21.23). Et, dans la Règle de la Communauté, "le transfert du besoin de pureté par rapport au Temple au besoin de pureté par rapport à la communauté est complet. Autour de la communauté se sont développés des cercles concentriques de pureté, comme autrefois autour du Saint des Saints. La pureté requise pour approcher le Temple est maintenant devenue nécessaire pour appartenir à la Communauté $»^{30}$. On peut dire ainsi que « la purification n'a plus lieu à l'intérieur du Temple ni à travers les sacrifices qui y sont offerts; elle s'obtient à l'intérieur de la communauté, le Temple nouveau dans lequel habite l'esprit de Dieu qui fait de la communauté un lieu de purification et de justification ${ }^{31}$. Les membres de la secte ainsi purifiés atteignent le même

[122] ${ }^{25}$ F. GARCÍA MARTíNEZ, « Les limites de la communauté : pureté et impureté à Qumrân et dans le Nouveau Testament », in T. BAARDA (éd.), Text and Testimony: Essays in Honor of A.F.J. Klijn, Kampen, Kork, $1988,111-122$.

26 Ibid., p. 114. Il voit cette tendance attestée notamment par 11Q19 45,11-12 qui interdit tout rapport sexuel au sein de la ville sainte. Il renvoie également à 45,7-10;46,16-18, 47,7-18;52,19-21, voire à 46,13-16.

27 Ibid. Cette tendance lui paraît plus particulièrement attestée par la disposition qui interdit aux aveugles l'accès de la ville sainte (11Q19 45,12-13), leur appliquant ainsi une règle qui ne visait dans le Pentateuque (Lv 21,18) que ceux d'entre les prêtres qui étaient aveugles et qui se trouvaient dès lors exclus du sacerdoce (p. 115). F. García Martínez relève qu' « une série de textes qumrâniens interdit l'appartenance à la secte [CD 11,15-17, qu'atteste désormais 4QDb], ou à la congrégation de l'époque eschatologique [1QSa 2,5-6], ainsi que la participation à la guerre finale [ainsi 1QM 7,3-6] aux mêmes personnes qui sont exclues des fonctions sacerdotales selon Lv 21,17-20 » (ibid.).

${ }^{28}$ Ibid. Cette tendance apparaît dans toute une série de dispositions majorant l'impureté résultant du contact avec un cadavre (11Q19 49,11-15; 50,10-1651,4-5).

[123] ${ }^{29}$ Ibid., p. 117.

30 Ibid., p. 118. Il renvoie notamment à 1QS 6,24-25.

31 Ibid., p. 119, qui cite, à l'appui de cette affirmation, 1QS 3,8-9. 
niveau de pureté que les anges et se trouvent en communion avec eux au sein même de la communauté ${ }^{32}$ dont la liturgie est elle-même associée à la liturgie céleste ${ }^{33}$.

Il y a, selon nous, dans ces diverses représentations, un préalable fondamental pour comprendre ce qui se produit avec Jésus et, ensuite, dans le Nouveau Testament. Le rapport à la pureté et à la sainteté s'avère en effet déterminant aux origines du mouvement chrétien. Il nous semble que, de ce rapport, l'Évangile selon Marc nous fournit une présentation profilée qui s'avère d'un intérêt tout particulier car elle permet de comprendre, comment, à partir de Jésus et en se fondant sur sa proclamation et sur son action, le mouvement chrétien naissant a été amené à réinterpréter la pensée du Temple.

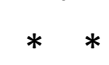

\section{[124]}

II. LE RAPPORT, NOUVEAU, À LA PURETÉ ET À L'IMPURETÉ DANS L'EVANGILE DE MARC, À L'AUNE DU CHAPITRE 1

La thèse qui a été proposée à la discussion lors du séminaire est la suivante : l'évangile selon Marc se prête à une lecture qui fait apparaître un Jésus qui, dans sa prédication comme dans son action, aurait, de façon cohérente, revisité et réinterprété les catégories du pur et de l'impur en promouvant une conception conquérante de la sainteté, ce qui se traduit tant dans sa pratique exorciste et thérapeutique que sur d'autres plans encore ${ }^{34}$.

Le prologue de l'évangile (Mc 1,1-13) est d'emblée révélateur. Il situe le lecteur sur un terrain où s'opère une subversion des catégories traditionnelles puisqu'il est indiqué que le baptême de Jean offre en fait, en marge du Temple, ce que le sanctuaire était censé être seul

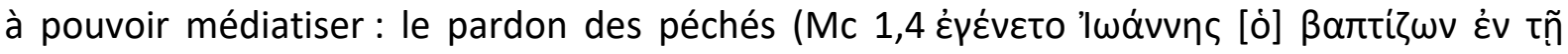

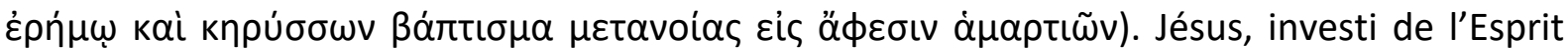
Saint dans le cadre de son propre baptême qui est couplé narrativement à l'Épreuve au désert, remporte une victoire décisive sur Satan lors de cette dernière. II rétablit ainsi l'harmonie originelle, ce que symbolisent la compagnie des bêtes sauvages et le service des anges (Mc $1,13)^{35}$. D'emblée, sa venue est placée ainsi dans une perspective [125] eschatologique, ce que

32 1QSa 2,3-11.

33 1QSb 4,24-27 et les Chants pour l'holocauste du Sabbat (4Q400-407 ; 11Q17).

[124] ${ }^{34}$ L'essai de NeYreY, The Idea of Purity (n. 5), et le commentaire de W.H. KelBer, Mark's Story of Jesus, Philadelphia, 1984, envisagent l'Évangile selon Marc dans une perspective qui trouvera ici de nombreux échos. Neyrey, p. 107, dresse ainsi une liste de quinze exemples dans lesquels le Jésus marcien paraît s'affranchir délibérément des règles de pureté. Les reprenant à son compte (pp. 390-391), D.B. DEPPE, The Theological Intentions of Mark's Literary Devices. Markan Intercalations, Frames, Allusionary Repetitions, Narrative Surprises, and Three Types of Mirroring, Eugene [Or], Wipf \& Stock, 2015, pp. 388-460, vient d'en proposer une très intéressante relecture et fait valoir que Marc croit que le Christ est devenu celui par lequel les Écritures d'Israël sont à la fois accomplies et correctement comprises (p. 458), ce qui fournit aux paganochrétiens une excellente raison d'abandonner les rites juifs. Pareille lecture est compatible avec la nôtre, même si l'accentuation n'est pas la même et si Deepe n'envisage pas, pour sa part, le lien du don de l'Esprit lors du baptême et de la défaite initiale subie immédiatement après par Satan au désert avec la suite du macro-récit. Il estime en fait que Marc recourt, dans les récits de controverses mettant Jésus face à des opposants, à des références ou à des citations scripturaires pour mieux montrer que, tout en prenant des libertés par rapport la Loi, Jésus en est en fait le fidèle interprète. L'explication qu'il propose peut fort bien être conçue en complément de celle que nous présentons ici et qui part du constat, réitéré par Jens SCHRÖTER, Jésus de Nazareth. A la recherche de l'homme de Galilée (Le Monde de la Bible 74), Genève, Labor et Fides, 2018, p. 156, avec Jésus, « la pureté était [...] "contagieuse" et prenait le pas sur l'impureté ».

35 Nous nous sommes employé ailleurs, GRAPPE, Baptême de Jésus et baptême des premiers chrétiens, in $R H P R 73,1993,377-393$, à montrer que les récits synoptiques du baptême de Jésus reposent sur une tradition qui mettait en œuvre une double typologie, adamique et messianique, et qu'ils contribuent [125] à 
vient confirmer le sommaire de sa prédication formulé en Mc 1,15 avec le double motif de l'accomplissement du temps fixé et de l'irruption du Royaume de Dieu.

Le reste du chapitre 1 illustre l'ampleur et les conséquences de la victoire remportée d'emblée sur Satan.

Le premier acte public de Jésus, après l'épisode de la vocation des premiers disciples (Mc 1,16-20), est l'exorcisme qu'il réalise le jour du sabbat alors qu'il enseigne dans la synagogue de Capernaüm (Mc 1,21-28). Cet épisode manifeste l'autorité particulière de Jésus dans son enseignement, un enseignement qui se distingue de celui des scribes précisément

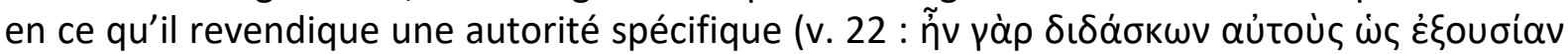

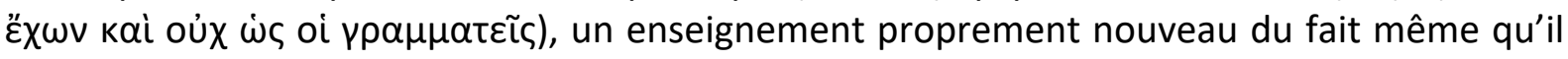

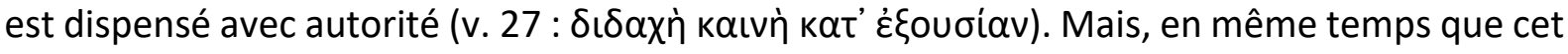
épisode [126] révèle l'autorité particulière de l'enseignement de Jésus, il illustre aussi le pouvoir qui est le sien. Il lui permet de venir à bout de l'esprit impur (v. 23.26) qui tourmentait

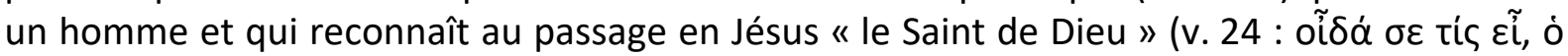
ä

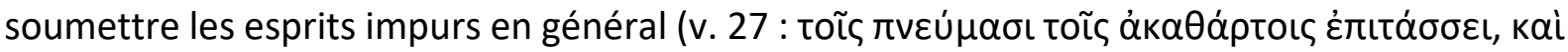

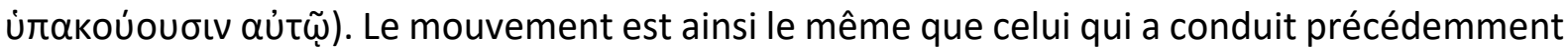
de la réception de l'Esprit au moment du baptême à l'issue victorieuse de l'épreuve au désert face à Satan. Celui qui est au bénéfice de l'Esprit (Saint) ${ }^{36}$ et que Dieu a désigné comme Son Fils apparaît comme le Saint de Dieu et maîtrise les puissances hostiles. Comme aux versets 14-15, l'évocation de la prédication de Jésus fait suite à celle du pouvoir dont l'a doté l'Esprit saint. Et le verdict de la foule, en Mc 1,27, associe, d'une manière étonnante, mais qui s'avère

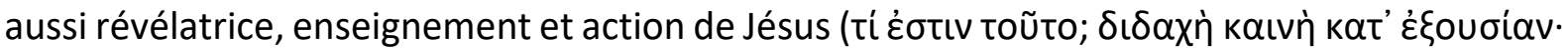

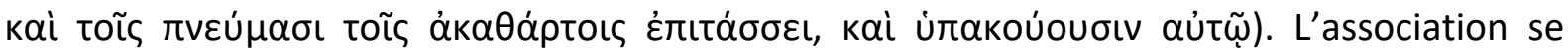
retrouve d'ailleurs en 1,39 où sont envisagées conjointement prédication publique et

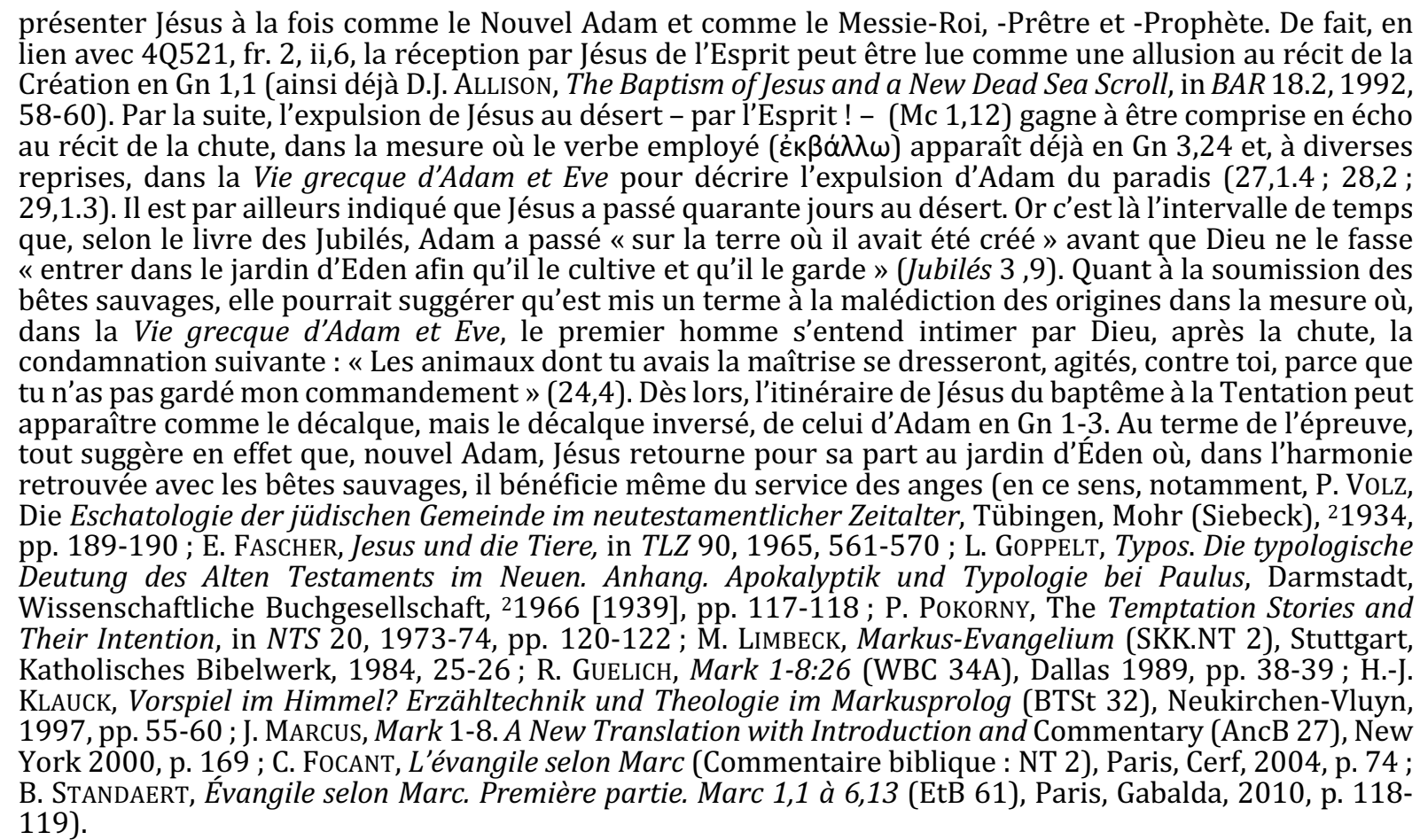

[126] ${ }^{36}$ Il n'est fait mention que de l'Esprit et pas de l'Esprit Saint au moment du baptême de Jésus, mais, dès lors que le Baptiste a annoncé en 1,8 que Jésus baptisera d'Esprit Saint, il est hautement vraisemblable que les deux formulations s'équivalent, ce que corrobore aussi la suite du récit, et plus particulièrement 1,24 . 


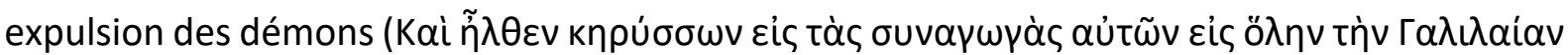

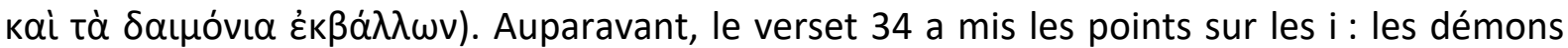
connaissent Jésus, ou le reconnaissent, et c'est la raison pour laquelle il ne les laisse pas parler

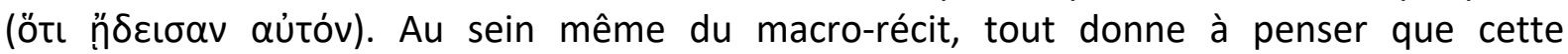
connaissance est liée au fait que, adjuvants de Satan, ils sont dans la confidence et savent qu'ils ont affaire à un adversaire redoutable qui est déjà sorti vainqueur de celui dont ils sont les suppôts, la victoire remontant à sa confrontation initiale avec lui ${ }^{37}$, ce que corrobore à sa manière $M c 3,11^{38}$.

[127] Juste après, Jésus guérit un lépreux, ou plus précisément le purifie (v. 41 et 42), non sans l'avoir préalablement touché (v. 41). Ici, on peut avoir l'impression que le rapport classique à l'impureté $s^{\prime}$ inverse ${ }^{39}$. Les lépreux étaient tenus, à partir des recommandations figurant en Lv 13-14, pour des sujets impurs par excellence. Non seulement Jésus ne craint pas de toucher l'un d'entre eux, mais, au moment même où il le touche, il lui ordonne d'être

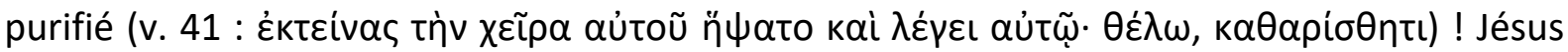
outrepasse en l'occurrence toutes les barrières, enfreint les cercles de pureté et vient établir cette pureté là même où l'impureté s'avérait jusque-là la plus contagieuse ${ }^{40}$. Dans la suite du macro-récit, il apparaîtra de manière récurrente que les malades $(3,10)$, comme la femme affectée d'une perte de sang $(5,27-30)$, cherchent à toucher Jésus pour être guéris $(5,28)$ ou que l'on prie Jésus de toucher certains d'entre eux pour les guérir $(8,22)$. II paraît clair que

37 On notera que, en Mc 1,31, au terme de la guérison de la belle-mère de Pierre (Mc 1,29-31), qui est elle-même insérée entre le récit de l'exorcisme dans la synagogue de Capernaüm (Mc 1,21-28) et le sommaire de Mc 1,32-33, qui associe guérisons en tout genre et, à nouveau, exorcismes, il est indiqué que la mère de Pierre, une fois guérie, se trouve vis-à-vis de Jésus et des disciples dans la même attitude que les

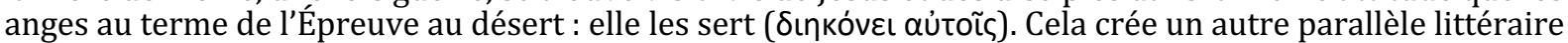
et thématique avec la complexe baptême de Jésus - épreuve au désert.

Le lien entre Mc 1,21-28 et Mc 1,9-13 est perçu à sa manière par A. YARBro-Collins, Mark. $A$ Commentary (Hermeneia), Augsburg, Fortress Press, 2007, p. 174, qui estime que, si les spectateurs de la scène ne comprennent apparemment pas le fait qu'autorité d'enseignement et pouvoir d'exorciser les démons ont une source divine, « le lecteur implicite (the audience) sait que cette autorité s'enracine dans la désignation de Jésus en tant que Messie et dans le don qui lui a été fait de l'Esprit Saint ». L'hypothèse de P.W. HollenBACH, The [127] Conversion of Jesus: From Jesus the Baptizer to Jesus the Healer, in ANRW II.25.1, Berlin, Walter de Gruyter, 1982, 196-219, selon laquelle ce serait la découverte, consécutive à son baptême, de ses pouvoirs thérapeutiques et exorcistes qui aurait convaincu Jésus de rejeter le mode de vie ascétique de Jean et de modifier, au profit d'une eschatologie réalisée, ses représentations dernières, cela dès lors que les guérisons présentes manifestaient que Dieu venait dès à présent visiter son peuple et rendaient, de ce fait, vain le baptême, établit certes un lien entre pratique exorciste et proclamation du Royaume, mais néglige le rôle dévolu à l'effusion de l'Esprit et au rapport, nouveau, à la pureté qu'elle instaure. Sans établir non plus de lien avec l'épisode du baptême de Jésus, G. vAN OYEN, Lire l'évangile de Marc comme un roman. Traduit du néerlandais par Michel Perquy (Le livre et le rouleau 38), Bruxelles, Lessius, 2005, p. 92, lit Mc 1,21-28 en continuité avec Mc 1,12-13 : «Le premier acte libérateur posé par Jésus se situe parfaitement dans la foulée de ce qu'il a vécu antérieurement dans le désert. Il s'est battu pendant quarante jours avec le Satan et... il a vaincu. Cette anecdote doit avertir d'emblée le lecteur que Jésus est soutenu par Dieu et qu'il sera en mesure de vaincre le mal ».

38 Dans ce verset, il est indiqué que les esprits impurs, quand ils voient Jésus, se jettent à ses pieds et s'écrient qu'il est le Fils de Dieu, autre façon de suggérer que la connaissance qui est la leur résulte de l'événement clé que représente, en lien avec l'épreuve de Jésus au désert, son baptême au Jourdain qui est précisément le moment auquel est associée la révélation de son identité de Fils de Dieu.

39 Th. KAzEN, Jesus and Purity Halakhah: Was Jesus Indifferent to Impurity? (CB.NT 38), Stockholm, Almquist \& Wiksell International, 2002, p. 127, relève ainsi que ce qui se produit ici «n'est pas en phase avec l'interprétation de la Loi qui prévalait ». Cela étant, le propos de Kazen est historique et ne prend pas en compte la dynamique propre de l'Évangile de Marc, ce qui l'empêche notamment d'établir un lien à la fois logique et narratif avec le diptyque constitué par les deux récits du baptême de Jésus et de l'épreuve au désert. Il reconnaît cependant, p. 337, en commentant Mc 5,1-20, que cet épisode «évoque une lutte de pouvoir eschatologique, dans laquelle l'exorciste vainc et détruits l'impureté démonique » et ajoute que « cela peut se comprendre en fonction de son statut pur et saint [il parle ici de Jésus] ou encore en fonction d'un pouvoir inhérent [au personnage] ou d'une autorité spirituelle ».

$4^{0} \mathrm{On}$ se souviendra ici notamment du fait que, dans le Rouleau du Temple, un espace à part est réservé aux lépreux, au même titre qu'aux femmes ayant leurs règles ou leurs couches et aux personnes atteintes d'écoulement, en marge du dernier cercle de pureté que distingue cet écrit (11Q19 48,14-17). 
tous ces passages font écho, d'une manière [128] ou d'une autre, à la guérison initiale du paralytique et s'inscrivent dans la même dynamique qu'elle.

Il pourra être utile de se souvenir ici que le concept de Royaume de Dieu s'était luimême développé en lien étroit avec le Temple. Ce dernier n'était-il pas le lieu tout désigné de la manifestation du Dieu-Roi en tant que palais et sanctuaire à la fois ? Le culte sacrificiel était ainsi censé assurer les conditions de possibilité de la communion avec le Dieu-Roi. Et cela en vertu d'une logique selon laquelle les rites de réparation ou d'expiation constituaient le préalable indispensable à cette communion qui s'épanouissait dans des repas établissant une forme de commensalité entre Dieu et Son peuple ${ }^{41}$.

La dynamique instaurée par la prédication et l'action de Jésus vient ainsi, dans l'Évangile selon Marc, apporter, indépendamment du sanctuaire, cela même que le culte du Temple était censé procurer : la communion du Royaume. De fait, la proclamation de l'avènement du Royaume du Dieu vient, ici et maintenant, investir la sphère profane. L'Esprit saint y est désormais à l'œuvre. II désenclave l'espace et le sanctifie ${ }^{42}$ ! Mais, alors que, dans le culte, dans la piété pharisienne et dans le vécu communautaire qumrânien, la réparation et les séparations apparaissaient comme des préalables indispensables à la communion, dans la proclamation par Jésus de l'avènement du Royaume, la communion est rendue immédiatement possible et court-circuite en quelque sorte la réparation.

\section{LE RAPPORT, NOUVEAU, À LA PURETÉ ET À L'IMPURETÉ DANS L'EVANGILE DE MARC ET LES CONSÉQUENCES QUI EN RÉSULTENT, À L'AUNE DE LA SUITE DU MACRO-RÉCIT}

Ce qui peut se vérifier à l'échelle de Marc 1, nous paraît trouver des prolongements dans la suite de l'œuvre.

La séquence narrative qui conduit de Mc 2,1 à 3,6 montre Jésus poser de nouveaux signes de l'irruption du Royaume de Dieu et de la bonne nouvelle qu'elle constitue, en continuant de déplacer les barrières, de revendiquer une autorité toute particulière pour ce faire et de susciter l'opposition résolue de milieux attachés aux modes de représentation traditionnels.

[129] Dans un premier temps, à l'occasion du récit de controverse lié à la guérison du paralytique de Capernaüm (Mc 2,1-12), il revendique d'avoir sur la terre, en tant que Fils de

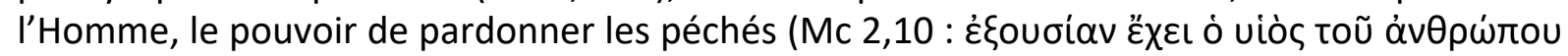

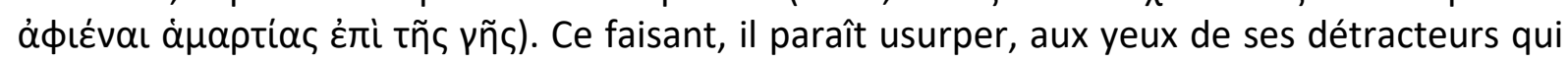
l'accusent de blasphème (Mc 2,7), une prérogative divine. Mais le pouvoir qu'il revendique en tant que Fils de l'Homme s'éclaire à la lumière des représentations qui, à partir de Dn 7,13, étaient venues s'associer à cette figure mystérieuse et sans doute collective à l'origine, mais qui n'a pas tardé à faire l'objet d'une interprétation individuelle et messianique, comme l'illustrent les Paraboles d'Hénoch (1 Hénoch 37-71) où s'opère la jonction entre la figure daniélique et celle de juge du monde. En 1 Hénoch, il est ainsi notamment dit que les élus mangeront en compagnie du Fils d'Homme $(62,14)$, conçu comme une figure céleste résidant en compagnie du Principe des jours, c'est-à-dire de Dieu en personne $(46,1-2)$, et que «la somme du jugement lui a été donnée » si bien que, à la fin des temps, « il fera disparaître les pécheurs de la face de la terre » $(69,27)$. Dans cette perspective, le fait que Jésus exerce en

[128] ${ }^{41}$ Nous avons amplement étayé la thèse énoncée dans ce paragraphe dans Ch. GRAPPE, $L e$ Royaume de Dieu. Avant, avec et après Jésus (Le Monde de la Bible 42), Genève, Labor et Fides, 2001, passim.

${ }^{42}$ Comme le note J.D.G. Dunn, Jesus and Holiness: The Challenge of Purity, in S.C. Barton (éd.), Holiness Past and Present, London - New York, T\&T Clark, 2003, p. 192 : «La sainteté était plus importante pour Jésus en tant que puissance qui nettoie ce qui est souillé et qui dissout l'impureté que comme statut (d'une personne ou d'un lieu) constamment menacé par ce qui est 'commun' et profane. » 


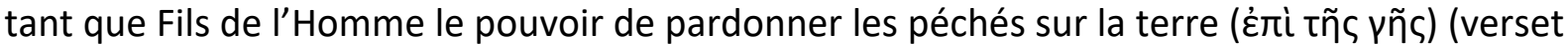

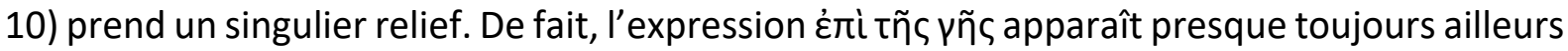

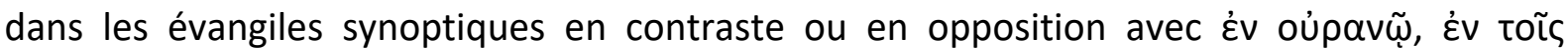
oủpavoĩ ou des expressions voisines (Mt 6,10. 19-20;16,19;18,19;23,9;28,18; LC 2,14). Or l'insistance est ici sur le fait que le Fils de l'Homme a autorité pour pardonner les péchés dès maintenant et ici-bas ${ }^{43}$. Sur l'arrière-plan des spéculations relatives au rôle eschatologique dévolu au Fils de l'Homme, on constate qu'il y a anticipation et délocalisation. Par ailleurs, et comme avec le baptême de Jean, la médiation du Temple se trouve ici occultée ou rendue vaine.

Dans la scène suivante, la vocation de Lévi (Mc 2,13-17), Jésus, après avoir appelé cet homme, collecteur d'impôt de son état, à le suivre et s'être attablé dans sa maison, s'entend

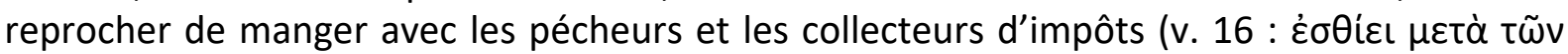

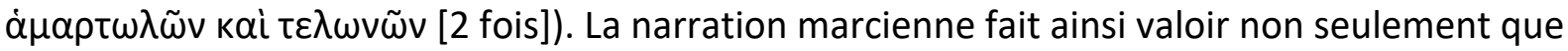
le Fils de l'Homme a pouvoir sur la terre de pardonner les péchés $(2,10)$ mais encore qu'il va à la rencontre des pécheurs et qu'il [130] s'agit là précisément de sa mission qui instaure une commensalité répréhensible aux yeux de ses adversaires. Cette mission consiste à appeler non

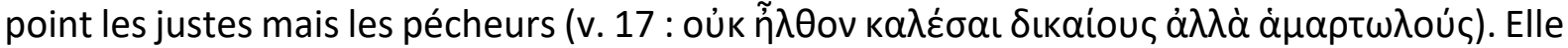
lui fait remplir un office comparable à celui d'un médecin qui ne s'adresse pas à ceux qui vont

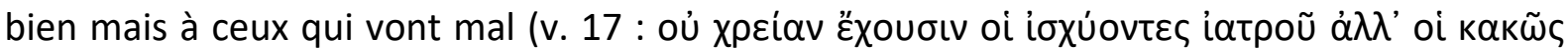

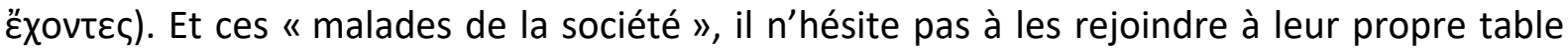
(v. 15-16), s'engageant ainsi dans une commensalité répréhensible aux yeux des scribes d'entre les pharisiens (v. 16), défenseurs résolus d'une pratique qui conduisait sans doute nombre d'entre eux à se placer à l'abri de leurs frères impurs au sein de haburoth.

La controverse relative au jeûne (Mc 2,18-22), pourrait bien fournir une nouvelle clé en vue d'une meilleure compréhension de l'attitude de Jésus. Aux disciples de Jean et aux (disciples des) pharisiens qui s'étonnent que les disciples de Jésus s'abstiennent de jeûner alors même qu'eux le font, il répond sous forme de question : " Les fils de la noce peuvent-ils

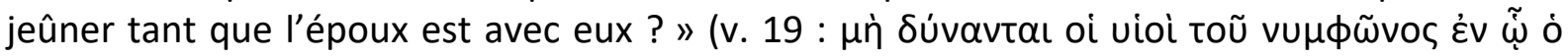

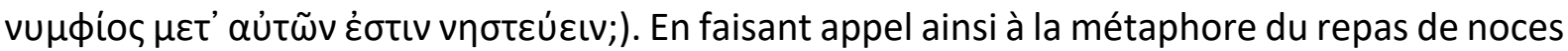
pour caractériser le temps de sa présence au milieu des siens, le Jésus marcien recourt de fait à deux images, celle du repas et celle des noces, particulièrement riches de sens dans la tradition du peuple d'Israël où elles avaient été valorisées tant dans une perspective historique qu'à I'horizon eschatologique ${ }^{44}$. En affirmant que sa présence au milieu des siens suspend le jeûne et en laissant entendre qu'elle inaugure une commensalité nouvelle parce que l'Époux se manifeste à travers lui au milieu des siens, il place son ministère sous un éclairage résolument eschatologique. La suite de la scène le confirme, qui envisage un retour au jeûne une fois que l'Époux aura été enlevé aux siens, c'est-à-dire au lendemain de la Passion et de la Résurrection (Mc 2,19b-20). Le ministère de Jésus apparaît ainsi d'un caractère tout à fait particulier. Pendant toute sa durée, c'est-à-dire tant qu' est immédiatement présent celui par lequel est proclamé l'avènement du Royaume de Dieu, il y a quasi-immédiateté dans

[129] ${ }^{43}$ Focant, L'évangile selon Marc (n. 35), p. 110, note ainsi, en commentant la première apparition du titre Fils de l'Homme dans l'œuvre : « Pour la première apparition de ce titre dans l'évangile, il est frappant de constater que le Fils de l'Homme n'apparaît pas sous les traits du juge eschatologique, comme en Dn 7 et dans le livre d'Hénoch, mais bien sous les traits de celui qui pardonne les péchés. Lui qui partage «l'autorité » ( $̇ \xi o u \sigma i \alpha$, Dn 7,14) de Dieu, il la fait venir sur la terre (Mc 2,10) sous les formes du pardon des péchés et de la guérison ».

44 Pour le festin eschatologique, voir notamment És 25,6-8 ; 1 Hénoch 62,14; 1QSa 2,17-21; 4Q521 ff. 2 II + 4 l. 13. Pour le motif des noces eschatologiques de Dieu avec Son peuple, Os 2,4-25; Es 54,4-8; 62,15 ; Jr 2,2-3 ; Éz 16. On notera encore que le thème des noces de Dieu avec son peuple, conçu dans la perspective de l'accomplissement dernier, a pu être envisagé en lien avec le motif du festin eschatologique. Tel est le cas en És 54,5-55,5 qui associe le motif des nouvelles noces de Dieu avec son peuple (54,6-10), de la Jérusalem nouvelle $(54,11-17)$, de l'invitation au banquet $(55,1-5)$ et du pèlerinage des nations $(55,5)$. 
les rapports avec Dieu. II n'y a plus [131] d'autre médiation nécessaire que la seule présence de Jésus. Et tous ceux qui le rencontrent sont invités au Festin ${ }^{45}$. Sans qu'il y ait besoin de rites préalables, la commensalité est possible avec celui qui annonce le pardon de Dieu. Plus tard, les médiations (rites) seront à nouveau nécessaires, mais là tout écart est supprimé entre Dieu et son Envoyé. On pourra noter à cet égard que les deux images qu'évoque la suite du discours de Jésus, celle du vieux vêtement et celle du vin nouveau (v. 21-22), s'inscrivent dans la même perspective d'inauguration d'un monde et d'un temps nouveaux qui requièrent de nouveaux cadres en faisant éclater les anciennes barrières et qui instaurent, de ce fait, un nouveau système ${ }^{46}$. La nature de l'argumentation peut être comparée à celle que l'on rencontrait déjà

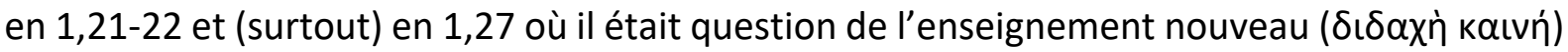
de Jésus, enseignement distinct de celui des scribes parce que dispensé avec autorité. Notre passage pourrait faire apparaître que cette autorité lui est conférée par l'irruption du temps nouveau des noces et du salut.

La controverse relative à la consommation des épis par les disciples de Jésus le jour du sabbat (Mc 2,23-28) et la controverse liée au récit de [132] la guérison de l'homme à la main sèche $(M c 3,1-6)$ ont toutes deux trait au sabbat. À travers ces deux récits est promue une compréhension nouvelle du jour du repos, ce que fait apparaître la comparaison que l'on peut établir avec la compréhension du sabbat qui prévalait tant en milieu pharisien qu'essénien ${ }^{47}$. Dans le premier des deux récits est affirmée la seigneurie du Fils de l'Homme sur le sabbat

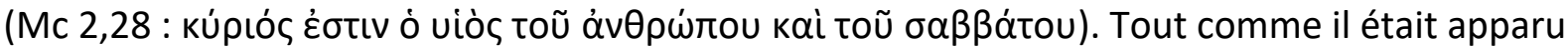
que le Fils de l'Homme avait pouvoir sur la terre de pardonner les péchés (v. 10), il apparaît désormais qu'il a autorité sur les temps qui, nous l'avons vu, étaient, tout comme les rites destinés à l'octroi par Dieu du pardon des péchés, soigneusement codifiés et distingués. Outre qu'il affirme l'autorité du Fils de l'Homme sur le sabbat, le Jésus marcien, dans ces deux scènes, invoque deux principes susceptibles de faire voler en éclat toute casuistique. II pose, en 2,27 , que "le sabbat est advenu pour l'homme et non l'homme pour le sabbat ». II

[131] ${ }^{45}$ J. Jeremias, Théologie $d u$ Nouveau Testament. Première partie. La prédication de Jésus (LeDiv76), Paris, Cerf, 1975, p. 149, écrivait ainsi que les repas pris par Jésus "sont des banquets eschatologiques, des prémices du festin de salut des derniers temps (Mt 8,11 par.), dans lesquelles est présente dès maintenant la communion des saints (Mc 2,19) ». Dans le même sens, J. BECKER, Jesus von Nazareth, Berlin - New York, Walter de Gruyter, 1995, p. 198, qui commente Mc 2,18-20 dans la même perspective que celle qui est ici la nôtre. De son côté, YARBRo-CoLlins, Mark (n. 37), p. 199, fait valoir que « le Jésus historique pourrait avoir revendiqué indirectement par ce logion être l'agent majeur de Dieu et médiatiser, de ce fait, la présence de Dieu dans les derniers jours ».

${ }^{46}$ L'image du vêtement peut être métaphore du monde, comme l'illustrent notamment És 51,6 et le Ps 101,26-28 (LXX), cité en He 1,10-12. On ne peut exclure, dans cette perspective, que la parabole de la pièce qui fait éclater le vieux vêtement contribue à faire valoir la radicale nouveauté du temps inauguré par la présence de l'Époux. Le monde se trouve renouvelé et la participation aux Noces requiert des habits neufs (dans cette perspective, voir notamment J. Jeremias, Les paraboles de Jésus [Livre de Vie 85-86], Le Puy, Xavier Mappus, 1962, p. 167, et E. HAulotTE, Symbolique du vêtement selon la Bible [Théologie 65], Paris, 1966, p. 321). Le vin nouveau est associé, dès le Premier Testament, au surgissement des temps nouveaux que Dieu inaugurera (Jl 2,19; Os 2,24 ; Za 9,17), même s'il est également évoqué, et plus fréquemment, dans le cadre de la vie quotidienne. À Qumrân, c'est le vin nouveau qui est, avec le pain, le composant essentiel du repas communautaire : « quand ils disposeront la table pour manger ou (prépareront) le vin pour boire, le prêtre étendra en premier sa main pour qu'on prononce la bénédiction sur les prémices du pain et du vin » (1QS 6,4-5). Ce repas communautaire était conçu lui-même comme la préfiguration du repas eschatologique qui serait présidé par les deux messies, royal et sacerdotal (Règle annexe de la Communauté,1QSa 2,17-21). La mention du vin nouveau peut donc évoquer ici les temps nouveaux, célébrés comme temps des noces et interprétés comme temps du salut (ainsi notamment JEREMIAS, Ibid., p. 168). Ce vin nouveau requiert d'être accueilli dans de nouveaux cadres, figurés ici par les outres neuves.

[132] ${ }^{47}$ La littérature pharisienne nous apprend qu'il était bel et bien proscrit de moissonner le jour du sabbat (Mishna Shabbat 7,2) et que l'hostilité des adversaires (pharisiens) de Jésus lors de la guérison de l'homme à la main sèche s'explique du fait qu'il n'y avait pas, en la matière, danger de mort (Mishna Yoma 8,6). La littérature essénienne rejoint la littérature pharisienne tout en affichant un rigorisme encore plus grand (Jubilés 50,6-13 et CD 9,13-14 où le cas, envisagé par Mt 12,11, du sauvetage d'une bête tombée dans un puits le jour du sabbat est exclu [il faut ajouter toutefois que l'attitude pharisienne en la matière est loin d'être aussi claire que ne semble le supposer le texte de Matthieu]). 
demande, en 3,4, s'il est permis, lors du sabbat, de faire le bien ou de faire le mal, de sauver une vie ou de tuer. Dans ce dernier cas, il subordonne une problématique rituelle à un impératif éthique ${ }^{48}$. Il peut paraître, en l'occurrence, extraire de la Loi un principe fondamental, l'amour du prochain, et refuser de l'étendre ou de le diluer ensuite en une multitude de réglementations spécifiques. Il se situe à l'opposé de la façon dont procédaient tant pharisiens qu'esséniens en élaborant justement une casuistique destinée à prévoir toutes les situations et à déterminer par avance la conduite à tenir dans chacune d'entre elles.

La première partie de notre parcours nous avait amené à rappeler que, à partir du Pentateuque et plus particulièrement des données encloses dans le Lévitique, s'était développée une pensée du Temple selon un ordre qui gravitait autour du Sanctuaire et du culte et qui supposait tout une série de distinctions entre les êtres, les animaux, les lieux et les temps.

[133] La lecture de Marc 2,1-3,6 peut donner à penser que, avec Jésus, cette pensée du Temple éclate ou se volatilise.

Il octroie le pardon des péchés en dehors du sanctuaire et indépendamment de tout rite destiné à le médiatiser. II va à la rencontre des pécheurs, les rejoint à leur table et suggère qu'ils ont part, là encore sans préalable ni rite, au Festin et à la commensalité du Royaume. II caractérise le temps de sa présence comme un temps totalement à part, qui suspend les rites précisément parce qu'il est temps de Noces. Il célèbre le sabbat comme un temps où se manifeste la volonté divine urgente de faire le bien et de sauver et balaie dès lors les distinctions patiemment et scrupuleusement établies.

La suite de la narration marcienne invite à poursuivre dans la même direction, comme nous nous emploierons à l'illustrer par quelques exemples.

Dès 3,7-8, l'origine des foules qui suivent Jésus fait apparaître une juxtaposition de personnes a priori juives (venues de Galilée, de Judée et de Jérusalem) et de personnes vivant à tout le moins dans un environnement païen (venues d'Idumée, d'au-delà du Jourdain, et des environs de Tyr et de Sidon, soit de la région-même dans laquelle Jésus rencontrera plus loin la femme syro-phénicienne). De manière proleptique, intérieur et extérieur des limites classiques d'Israël semblent ici se superposer, cela sans doute pour préparer le lecteur à la suite du macro-récit qui fera singulièrement bouger les lignes en matière de limites et de frontières ${ }^{49}$.

La controverse relative à Beelzeboul (Mc 3,22-30) joue un rôle clé dans l'argumentation marcienne du fait des correspondances qui apparaissent entre cette scène et le début de l'œuvre, le prologue complété par les versets 14-15 au caractère programmatique affirmé50. Ses adversaires accusent Jésus d'expulser les démons par le Prince des démons $(3,22)$, ce à quoi Jésus rétorque que Satan - qu'il a vaincu au terme de l'épreuve au désert $(1,13)$ - ne saurait être en lutte contre lui-même $(3,23)$, de même qu'un royaume - et l'on pense ici immédiatement au Royaume de Dieu qui a désormais fait irruption $(1,15)$ - ne saurait être divisé contre lui-même $(3,24)$. Et quand Jésus proclame que " personne ne peut, étant entré

48 Focant, L'évangile selon Marc (n. 35), p. 133, relève ainsi que « le sabbat fonctionne différemment selon qu'on se place dans la logique du règne de Dieu annoncé par Jésus ou qu'on la récuse. Pour les pharisiens, le sabbat constitue "un marquer identitaire", tandis que Jésus veut le visualiser comme lieu de la volonté divine que l'homme soit sauvé ».

[133] ${ }^{49}$ É. CuviLLIER, L'évangile de Marc (Bible en face), Paris - Genève, Bayard - Labor et Fides, 2002, p. 72, signale ainsi que «c'est la préfiguration de la portée universelle de l'Évangile qui est peut-être suggérée par la mention des régions avoisinantes ». Dans le même sens, FocANT, L'évangile selon Marc (n. 35), p. 140 (avec références bibliographiques complémentaires).

50 Sur ces correspondances et leur importance pour la compréhension de l'œuvre tout entière, voir déjà NEYREY, The Idea of Purity (n. 5), pp. 110-111. 


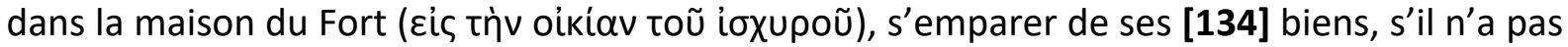
d'abord lié le Fort (tòv ioxupóv), et [que ce n'est qu'] alors [qu'] il s'empare de sa maison » $(3,27)$, le lecteur est invité à se souvenir que, en 1,7 , Jean a annoncé la venue du « plus fort

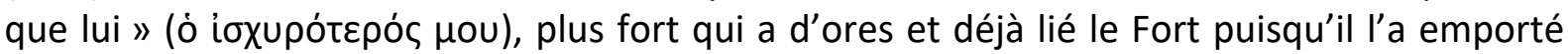
face à lui au désert. Alors que les esprits impurs n'ont pas tardé à reconnaître en Jésus « le Saint de Dieu » $(1,24)$, puis " le Fils de Dieu » $(3,11)$ - ce qui peut confirmer le lecteur dans la conviction qu'ils sont bien dans le secret de ce qui s'est joué lors du diptyque baptême Épreuve au désert - des autorités - en l'occurrence des scribes venus de Jérusalem - viennent remettre en question l'autorité du Maître $(3,22)^{51}$. La mention de la provenance de ces opposants pourrait être ici hautement symbolique ${ }^{52}$ : ils viennent du lieu même à partir duquel sont définis les cercles de pureté et s'inquiètent des agissements d'un franc-tireur qu'ils situent manifestement en dehors du système. On ne saurait s'étonner qu'ensuite Jésus stigmatise le blasphème contre l'Esprit Saint $(3,29)$, car c'est précisément doté de cet Esprit qu'il a vaincu Satan au désert et que, désormais reconnu comme le Saint de Dieu $(1,24)$ par les esprits impurs, il les met en déroute. En remettant en question son activité exorciste et en l'imputant à Satan et non à l'Esprit Saint, on se place résolument dans le camp de l'adversaire et on s'exclut de la dynamique générée par l'effusion de l'Esprit Saint et l'irruption du Royaume. Le macro-récit marcien s'avère ainsi d'une remarquable cohérence.

Dans la suite de la narration marcienne, Jésus va continuer de manifester son autorité sur les esprits impurs $\left(5,1-20^{53} ; 7,24-30^{54} ; 9,14-29\right)^{55}[135]$ et va d'ailleurs s'employer à étendre cette autorité à ses disciples en la leur transmettant $(6,7)$. L'application que met l'auteur à parler d'esprits impurs ${ }^{56}$ quasiment aussi souvent que de démons ${ }^{57}$, terme beaucoup plus usuel, illustre un souci de bien manifester qu'avec Jésus, le Saint de Dieu $(1,24)$, une forme de sainteté à la fois conquérante et purificatrice, est à l'œuvre ${ }^{58}$.

Au chapitre 7, le récit de controverse sur le pur et l'impur $(7,1-23)$ culmine au verset 15 avec le logion de Jésus suivant : « il n'y a rien d'extérieur à l'homme qui, entrant en l'homme, peut le souiller, mais ce qui sort de l'homme, c'est là ce qui souille l'homme ". Le commentaire explicite de l'évangéliste laisse clairement entendre qu'ainsi Jésus déclarait purs tous les

[134] 51 Comme le note CuviLliER, L'évangile de Marc (n. 49), en effectuant un parallélisme antithétique avec Mc 1,24 et 3,11, ils « ont de Jésus une opinion aussi affirmée que celle des esprits impurs mais diamétralement opposée ».

52 C'est ce que reconnaît à sa manière NEYREY, Ibid., p. 109.

${ }^{53}$ Ce micro-récit, au cours duquel l'homme possédé d'un esprit impur le reconnaît à son tour comme «Fils du Dieu Très-Haut» (Mc 5,7), conduit Jésus à réduire "Légion » au silence d'une manière aussi originale que radicale suite à leur noyade avec les porcs dans lesquels Jésus les a fait entrer en exauçant leur propre vœu ! Il marque aussi une première étape dans un processus qui va conduire Jésus à manifester que les effets de l'effusion de l'Esprit Saint et de la proclamation du Royaume ne se trouvent pas limités par les frontières d'Israël et peuvent se manifester au delà. Le démoniaque une fois guéri se lance d'ailleurs dans une entreprise d'annonce et de proclamation de ce que le Seigneur a fait pour lui, au-delà des frontières d'Israël et au sein du territoire qui est le sien, la Décapole.

54 À l'occasion de ce nouveau micro-récit qui se déroule en territoire païen, c'est la parole de la femme syro-phénicienne qui déclenche la guérison de sa fille possédée jusque-là par un esprit impur.

55 Dans le même temps, la menace de Satan continue de planer $($ Mc 4,15$)$ et peut même émaner des disciples eux-mêmes, et plus particulièrement du premier d'entre eux (Mc 8,33).

On pourra noter par ailleurs que le récit de la tempête apaisée (Mc 4,35-41) revêt lui-même les traits d'un exorcisme, dans la mesure où Jésus y réprimande le vent et lui adresse, [135] comme à l'esprit impur du démoniaque de la synagogue de Capernaüm auparavant (Mc 1,25), une consigne de silence (Mc 4,39).

${ }^{56}$ Mc 1,23.26.27 ; 3,11.30 ; 5,2.8.13 ; 6,7 ; 7,25; 9,25. De ces occurrences, seules 3,11; 5,13; 6,7 sont au pluriel.

${ }^{57}$ Mc $1,34.34 .39 ; 3,15.22 .22 ; 6,13 ; 7,26.29 .30 ; 9,38$. De ces occurrences, seules celles du chapitre 7 (épisode de la Syro-phénicienne) sont au singulier.

On peut inférer des observations faites ici et à la note précédente que Marc paraît privilégier, au pluriel, l'usage du mot démon et, au singulier, celui de la circonlocution esprit impur.

58 Dans le même sens J.H. NeYREY, The Idea of Purity (n. 5), p. 111, dont nous prolongeons ici quelque peu le propos. 
aliments $(7,19)$, abolissant de la sorte les règles de pureté alimentaires et pas seulement les dispositions pharisiennes au terme desquelles il convenait de se laver les mains ${ }^{59}$.

Au chapitre 11, par son intervention au Temple (Mc 11,15-18) ${ }^{60}$, Jésus s'en prend aux marchands et aux changeurs qu'il expulse (v. 15) comme auparavant il a expulsé les démons (voir notamment Mc 1,39.43; 6,13). [136] Ces marchands et changeurs remplissaient, de par leur activité, une fonction d'intermédiaires obligés en vue de la célébration du culte puisqu'ils permettaient l'échange d'une monnaie profane contre une monnaie pure ou contre des bêtes rituellement pures ${ }^{61}$. Jésus peut même paraître entraver la bonne marche du culte sacrificiel et provoquer son interruption en empêchant la circulation de tout vase (v. 16) ${ }^{62}$, dans la mesure où le terme бкعṽoৎ est un terme technique désignant fréquemment les ustensiles cultuels $^{63}$. La suspension implicite du culte qui est ainsi suggérée nous situe à nouveau sur le plan de la subversion de l'institution du culte et des mécanismes de réparation et de communion qu'elle met en œuvre ${ }^{64}$.

À la suite de son intervention au sanctuaire, Jésus est interrogé sur l'autorité en vertu de laquelle il agit (Mc 11,27-33) et, comme par hasard, il renvoie au baptême de Jean, un baptême doté lui-même d'une portée subversive puisque, conçu, nous l'avons dit, comme un baptême en vue du pardon des péchés (Mc 1,4), il pouvait se présenter comme un rite dispensé en marge du Temple - au Jourdain - tout en procurant ce que le culte au Temple luimême était censé procurer, la réparation ${ }^{65}$. Le fait que le Nazaréen précise implicitement que ce baptême de Jean pouvait se recommander d'une autorité en provenance du ciel et non pas des hommes $(11,30)$ vient faire écho au récit du baptême de Jésus, à l'occasion duquel une voix en provenance du ciel vient précisément asseoir Jésus dans le statut et l'autorité qui lui permettent d'œuvrer comme il le fait tout au long de l'œuvre.

Au cours de son procès, des témoins accusent ensuite Jésus de s'être fait fort de détruire ce sanctuaire fait de main d'homme et d'en rebâtir un autre, non fait de main d'homme, en trois jours (Mc 14,58).

Enfin, au moment de sa mort, dans ce qui constitue une remarquable inclusion avec le début de l'œuvre, le rideau du Temple, dont il faut se [137] souvenir qu'il était revêtu d'une

${ }^{59}$ L'ablution des mains avant les repas a, selon toute vraisemblance, une origine cultuelle (Ex 30,1821). La pureté n'était requise à l'origine que pour les repas pris par les prêtres et composés de viande offerte en sacrifice ( $\mathrm{Nb}$ 18,8-13). Mais cette exigence concernant les officiants dans l'enceinte du sanctuaire fut étendue, en milieu pharisien, à la vie quotidienne (Mishna Hagiga 2,5: « On se lave les mains pour manger des nourritures profanes ; mais pour les prélèvements [sacerdotaux], pour les dîmes et pour les nourritures sacrées, on doit se baigner... »). Toute nourriture était susceptible d'être traitée comme si elle était offerte dans le Temple (Talmud de Babylone Hullin 105a ; 106ab ; Shabbat 13b-14b). Quant à la réglementation complexe relative au lavement des mains, elle fit l'objet de tout un traité de la Mishna (Yadaim : voir notamment 1,1 et 2,3, passages qui traitent de la quantité d'eau requise en pareille circonstance. Cette quantité pouvait être faible et tenir dans le poing !). La même extension des requêtes visant les seuls prêtres était de mise en milieu essénien. Ainsi, à Qumrân, un bain d'immersion, soit davantage encore que la seule ablution des mains, était-il pratiqué par chacun des membres de la secte avant tout repas (cf. BJ II, 129132).

60 Pour plus de précisions sur ce texte et sur sa portée, nous renverrons à notre contribution Jésus, le Temps et les temps. À la lumière de son intervention au Temple, in Ch. GRAPPE - J.-C. INGELAERE (éds) Le Temps et les Temps dans les littératures juives et chrétiennes au tournant de notre ère (SJSJ 112), Leiden, Brill, 2006, 169-182.

[136] ${ }^{61}$ Ainsi K. Paesler, Das Tempelwort Jesu. Die Traditionen von Tempelzerstörung im Neuen Testament (FRLANT 184), Göttingen, Vandenhoeck \& Ruprecht, 1999, p. 247.

62 Ainsi W. SchenK, Der Passionsbericht nach Markus. Untersuchungen zur Überlieferungsgeschichte der Passionstradition, Gütersloh, Mohn, 1974, 155-157, que suivrait volontiers J. GNILKA, Das Evangelium nach Markus. 2. Teilband. Mk 8,27-16,20, Zürich - Neukirchen, Benzinger - Neukirchener, 1979, p. 129. Ainsi aussi Ch. PERrot, Jésus et l'histoire (CJJC 11), Paris, Desclée, 1979, p. 147.

63 Tel est le cas dans la Septante, mais aussi dans les littératures intertestamentaire $(2 \mathrm{Ba}$ 6,7-10 ; 80,2 ) et rabbinique (traité Kelim de la Mishna consacré aux objets) et chez Flavius Josèphe, BJ I,39.

${ }^{64} \mathrm{~A}$ ce sujet, nous nous permettons de renvoyer à notre contribution Jésus, le Temps et les temps (n. 54).

65 Dans le mêm sens, G. THEISSEN, Jésus et Jean Baptiste - Rupture ou continuité, in Andreas DETTWILLER (éd.), Jésus de Nazareth. Études contemporaines (Le monde la Bible 72), Genève, Labor et Fides, 2017, p. 83. 
symbolique cosmique ${ }^{66}$, se déchire. L'épisode vient faire écho sur plusieurs points à la scène du baptême ${ }^{67}$, deux parallèles apparaissant plus particulièrement frappants : une déchirure (du ciel ou du rideau du Temple) suggérant un bouleversement cosmique se produit et s'accompagne de la proclamation de la qualité de Fils de Dieu de Jésus.

Le récit fait ainsi valoir, en écho à la scène du baptême et au moment où ec-spire $(15,39)$ celui qui avait été investi par l'Esprit Saint au Jourdain $(1,10)$, que la séparation du Saint (ou du Saint des Saints) est abolie au moment même où cet homme, en qui il convient de reconnaître le Fils de Dieu, meurt. Cela manifeste que Dieu fait disparaître, précisément à ce moment-là, la distance et la frontière entre, d'une part, Sa gloire et Sa sainteté et, d'autre part, celui qui vient de mourir dans la détresse. Cela suggère en même temps que le lieu de rencontre avec Dieu passe désormais non plus par le Temple mais par le Fils de Dieu crucifié ${ }^{6}$.

\section{Conclusion et Prolongement}

Au terme du parcours qui aura été le nôtre, il nous semble ainsi possible de proposer que l'Évangile selon Marc puisse ainsi être lu dans son ensemble comme un écrit faisant valoir de façon cohérente comment la venue de Jésus inaugure, à partir du diptyque constitué par le récit de son [138] baptême (et de l'effusion de l'Esprit Saint qui l'accompagne) et par le récit de l'épreuve au désert (et de la victoire sur Satan qui la conclut), une ère nouvelle en même temps qu'un rapport nouveau à la sainteté et à l'impureté, aux lieux et aux temps, aux hommes et à Dieu.

En guise de prolongement de cette enquête, il nous semble que l'on peut relever une parenté fondamentale entre cette perspective marcienne et le logion relatif à l'irruption du Royaume de Dieu en lien avec l'expulsion des démons que l'on trouve dans la source $Q$.

De fait, dans ce logion, les guérisons et exorcismes de Jésus apparaissent comme des signes de l'irruption du Royaume ici et maintenant ${ }^{69}$.

Mt 12,28

Et si (c'est) par l'Esprit de Dieu que moi j'expulse les démons alors le Règne (ou le Royaume) de Dieu est arrivé jusqu'à vous
Lc 11,20

Et si (c'est) par le doigt de Dieu que [moi] j'expulse les démons alors le Règne (ou le Royaume) de Dieu est arrivé jusqu'à sur vous

[137] ${ }^{66}$ Flavius Josèphe et Philon d'Alexandrie décrivent l'un et l'autre les deux rideaux du Temple (BJ V,212-213; De vita Mosis II,88) : le rideau extérieur dont les couleurs symbolisent les quatre éléments $(B J \mathrm{~V}, 212-213$; Mos. II,88) et dont les dessins évoquent la voûte céleste $(B J \mathrm{~V}, 214)$; le rideau intérieur qui sépare symboliquement le ciel et la terre $(A J$ III,123) ou le monde qui change et celui qui ne change pas (Philon, Quaestiones in Exodum. II,91). On constatera que cette symbolique est relativement semblable. Elle peut s'expliquer en fonction du point de vue que l'on adopte : regard porté sur l'édifice (conçu comme lieu de la rencontre si problématique entre les hommes et Dieu) de l'extérieur ou de l'intérieur (du point de vue de l'homme ou de Dieu). Cette symbolique paraît fort ancienne. C'est ce que corrobore Si 50,5-7 (environ 180 avant notre ère).

67 On notera que, chez Marc, le verbe $\sigma x i \zeta \omega$, qui est employé à son propos, n'apparaît qu'à un autre endroit, pour signifier justement la déchirure des cieux, au moment du baptême de Jésus $(1,10)$. Cette déchirure est elle-même immédiatement suivie par la proclamation divine relative à la qualité de Fils de Dieu de Jésus $(1,11)$. Or cette proclamation, répétée devant les trois témoins privilégiés lors de la Transfiguration $(9,7)$ et qui doit être tue jusqu'à la résurrection $(9,9)$, trouve au Golgotha un écho dans la confession de foi du centurion $(15,38)$. Enfin Jésus, qui avait vu l'Esprit descendre sur lui au moment de son baptême $(1,10), e c$-spire à présent, rendant à présent au Père l'Esprit dont il l'avait investi.

68 R. FELDMEIER, Der Gekreuzigte im “Gnadenstuhl”. Exegetische Überlegungen zu Mk 15,37-39 und deren Bedeutung für die Vorstellung der göttlichen Gegenwart und Herrschaft (WUNT 69), in M. PHILONENKO (éd.), Le Trône de Dieu/Das Thron Gottes, Tübingen, Mohr Siebeck,1993, 213-232.

[138] ${ }^{69}$ Ainsi B. Chilton, Pure Kingdom. Jesus' Vision of God (Studying the Historical Jesus), Grand Rapids, Eerdmans, 1996. p. 73, qui renvoie aussi sur le même thème au logion 86 de l'Évangile selon Thomas : "Jésus dit : "Celui qui est près de moi est près du feu, et celui qui est loin de moi est loin du Royaume" ». 
Les deux passages ne divergent que par l'instrument de l'expulsion des démons, à savoir I'Esprit de Dieu ou le doigt de Dieu, et il est tout à fait vraisemblable que la forme primitive du logion nous ait été conservée par l'auteur à Théophile ${ }^{70}$.

La mention du doigt de Dieu renvoie à un passage bien précis de l'Écriture, Ex 8,15. C'est là en effet le seul endroit où il est question du doigt de Dieu dans le Premier Testament en tant qu'instrument de Sa puissance. Il s'agit du récit de la troisième plaie d'Egypte, à l'occasion de laquelle, pour la première fois, Moïse et Aaron infligent au pays une plaie, celle des moustiques ou de la vermine, que les magiciens n'arrivent pas à reproduire par leurs sciences occultes. La situation inspire aux magiciens le commentaire suivant : " C'est le doigt de Dieu !" $($ Ex 8,15).

Le logion de Jésus suffit ainsi à suggérer que, en lui, se manifeste une force agissante comparable à celle qui, jadis, a permis, avec Moïse et Aaron, la libération du pays d'Égypte ${ }^{71}$.

Mais il y a plus. Le contexte dans lequel s'insère ce logion est commun à Matthieu et Luc. Ils présentent ainsi au même endroit une commune addition par rapport au texte de Marc. Cela plaide en faveur du [139] ferme ancrage de ce logion, dans la tradition, au passage dans lequel il prend place, la controverse relative à Jésus et Béelzéboul (Mc 3,22-30 // Mt 12,22-32 et Lc 11,14-23). Le rapprochement avec l'Exode n'en devient dès lors que plus frappant puisque, dans les deux cas, on a affaire à une controverse relative à l'origine d'un acte merveilleux, que cet acte relève du miracle ou de l'exorcisme. L'activité exorciste de Jésus se trouve ainsi valorisée, au plus haut point, et par lui-même ${ }^{72}$.

Cela peut plaider en faveur de l'hypothèse selon laquelle Marc, tout en ne faisant pas état de ce logion, a compris le ministère de Jésus d'une manière qui se situe dans le droit fil de sa proclamation de l'irruption du Royaume. Une irruption du Royaume que les exorcismes et le rapport nouveau à la pureté qui s'instaure viennent attester.

70 Nous nous permettons ici de renvoyer à notre étude, Chr. GRAPPE, Jésus exorciste à la lumière des pratiques et des attentes de son temps, in Revue Biblique 110, 2003, 178-196.

71 Ibid.

[139] ${ }^{72}$ De son côté KAZEN, Jesus and Purity (n. 39), p. 337, écrit : « Je serais enclin à suggérer que Jésus et ses disciples considéraient ce pouvoir ou cette autorité [se manifestant dans les exorcismes], le "doigt de Dieu", comme un signe, ou même, comme un résultat du Royaume en marche (a result of the coming Kingdom). Si Jésus a compris son autorité comme le pouvoir du Règne de Dieu en marche, en quelque sorte incorporé en lui, ou encore résidant en lui, cela pourrait expliquer sa pratique exorciste singulière, qui ne repose pas sur des mesures de précautions et des techniques externes ». 